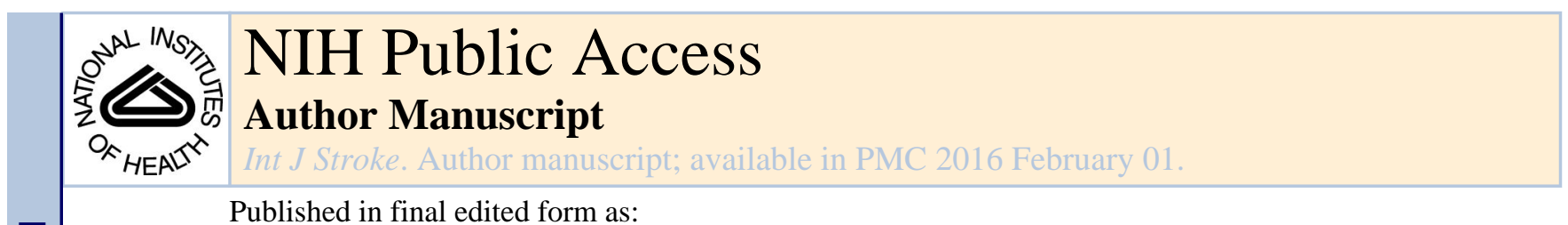

Published in final edited form as:

Int J Stroke. 2015 February ; 10(2): 194-201. doi:10.1111/j.1747-4949.2012.00919.x.

\title{
Thromboelastography in Patients with Acute Ischemic Stroke
}

Andrea Elliott, MD ${ }^{1}$, Jeremy Wetzel, BA ${ }^{1}$, Tiffany Roper, BA ${ }^{1}$, Evan Pivalizza, MBChB ${ }^{1}$, James McCarthy, MD ${ }^{1}$, Cristina Wallace, MD1 , Mary Jane Hess, BSN ${ }^{1}$, Hui Peng, PhD $^{2}$, Mohammad H Rahbar, PhD², Navdeep Sangha, MD1, and James C Grotta, MD'

${ }^{1}$ Department of Neurology, The University of Texas Medical School, Houston, TX

${ }^{2}$ Center for Clinical and Translational Sciences, University of Texas at Houston, Houston, TX

\begin{abstract}
Background-Thromboelastography (TEG) measures the dynamics of coagulation. There are limited data about TEG in acute ischemic stroke other than a single study from 1974 suggesting that acute ischemic stroke patients are hypercoagulable. There have been no studies of TEG in the thrombolytic era despite its potential usefulness as a measure of clot lysis. This study was designed to provide initial TEG data in stroke patients before and after tissue plasminogen activator (tPA) therapy, and to provide the necessary preliminary data for further study of TEG's ability to identify clot subtype and predict response to tPA therapy.
\end{abstract}

Methods-All acute ischemic stroke patients presenting between 11/2009 and 2/2011 eligible for tPA therapy were screened and 56 enrolled. Blood was drawn before (52 patients) and 10 minutes after tPA bolus (30 patients). Demographics, vitals, labs, 24hr National Institutes of Health Stroke Scale (NIHSS) and computed tomography (CT) scan results were collected. Patients were compared to normal controls.

Results-Acute ischemic stroke patients had shorter $\mathrm{R}(4.8 \pm 1.5$ vs $6.0 \pm 1.7 \mathrm{~min}, \mathrm{p}=0.0004)$, greater a-Angle ( $65.0 \pm 7.6$ vs $\left.61.5 \pm 5.9^{\circ}, \mathrm{p}=0.01\right)$, and shorter $\mathrm{K}(1.7 \pm 0.7$ vs $2.1 \pm 0.7 \mathrm{~min}, \mathrm{p}$ $=0.002$ ) indicating faster clotting. Additionally, a subset formed clots with stronger platelet-fibrin matrices. Treatment with tPA resulted in reduction in all indices of clot strength (LY30 $=0(0-0.4)$ vs $94.4(15.2-95.3) \mathrm{p}<0.0001)$, however there was considerable variability in response.

Conclusions-TEG demonstrates that many acute ischemic stroke patients are hypercoaguable. TEG values reflect variable clot subtype and response to tPA. Further study based on these data will determine if TEG is useful for measuring the dynamic aspects of clot formation and monitoring lytic therapy.

\section{Keywords}

cerebral infarction; ischaemic stroke; thrombolysis; stroke; tPA; haemocoagulation

Corresponding Author: James C. Grotta, MD, The University of Texas Medical School at Houston, Department of Neurology, 6431 Fannin, MSB 7.044, Houston, TX 77030, Phone: 713.500.7088, Fax: 713.500.0660, james.c.grotta@ uth.tmc.edu.

The authors report no conflicts of interest. 


\section{BACKGROUND}

The cornerstone of current therapy for acute ischemic stroke (AIS) is based on lysis of an offending blood clot, yet studies of clot formation or its response to lysis are relatively sparse. Thromboelastography (TEG) measures the dynamics of clot formation, strength, and lysis, and may also provide information about clot consistency. Therefore, TEG might be useful for assessing coagulation status and response to thrombolytic therapy.

TEG has been in use since 1948 but only recently has practical application been feasible after the advent of user-friendly computerized analysis. One key advantage of TEG is the global view it provides of the entire coagulation process ${ }^{1}$. Tests such as bleeding time, prothrombin time (PT), international normalized ratio (INR), partial thromboplastin time (PTT), thrombin time, fibrinogen and coagulation factor assays are all based on a static endpoint ${ }^{2}$. TEG provides integrated information on the balance between the two components of coagulation, thrombosis and lysis, measuring the dynamic coagulation process from initial clotting cascade to clot strength ${ }^{2}$.

Measurements are recorded as a thromboelastogram with the following parameters (Fig 1). SP (minutes) is the time elapsed from when the blood is placed in the TEG device until the first fibrin formation is detected, and $\mathrm{R}$ is the time until clot firmness reaches an amplitude of $2 \mathrm{~mm}$. R-SP, or delta (minutes), is the time to reach top speed of initial clot formation, reflecting the thrombin "burst". Anticoagulants will prolong R and delta and hypercoagulable states will shorten their duration. $\mathrm{K}$ is measured from $\mathrm{R}$ until clot firmness is $20 \mathrm{~mm}$ and measures of speed of clot strengthening. a Angle is formed by the slope of the TEG tracing at $\mathrm{R}$ from the horizontal; a Angle also reflects the speed of clot strengthening. Maximum Amplitude (MA) is the maximum strength of the clot, and $\mathrm{G}$ (dynes $/ \mathrm{cm}^{2}$ ) measures clot firmness, or strength $(\mathrm{G}=5,000 \mathrm{MA} /(100-\mathrm{MA}))$. G values are higher in clots that are more platelet rich and are held together by stronger fibrin matrices. Clot lysis is measured as the decay in MA over 30 minutes (LY 30), reported as percent lysis.

Currently there is great interest in the clinical applications of TEG $^{3-12}$. Most pertinent is a single study from 1974 before the test became automated. In this study TEG identified a hypercoagulable state in $29-38 \%$ of patients with an ischemic stroke versus $12 \%$ of agematched controls ${ }^{13}$. While other studies have also shown relative hypercoagulability of AIS patients using other methods, such as D-dimer ${ }^{14-17}$, no studies of TEG or other coagulation measures have related the coagulation status of patients in the hyperacute stage of stroke or with the use of lytic therapy. Currently tPA is given as a standard dose determined by the patient's body weight. Given the variability in achieving clot lysis and arterial recanalization following tPA ${ }^{18,19}$, it is possible that this dose is sometimes too small or too large leading to either incomplete thrombolysis or bleeding, respectively.

\section{AIMS AND HYPOTHESES}

Therefore, in this first exploratory study of TEG in AIS patients who were candidates for tPA therapy, we aimed to address the following hypotheses: 
1. The distribution of TEG values will reflect hypercoagulability in AIS patients compared to controls.

2. The distribution of TEG values will vary among AIS patients, particularly $G$ values which reflect the strength of the fibrin-platelet matrix.

3. The distribution of TEG values obtained post-tPA treatment will reflect variable degrees of fibrinolytic activity.

If these hypotheses could be confirmed, the data would then be used to inform the sample size needed for subsequent studies to address important clinical questions such as whether the baseline or post-tPA values will reflect clot subtype and predict the likelihood of recanalization or bleeding after tPA. Ultimately, the goal of our work is to determine if TEG can be useful to either adjust the dose of tPA or plan adjunctive therapy.

\section{METHODS}

This study was approved by the Committee for the Protection of Human Subjects (CPHS) of The University of Texas Health Science Center at Houston.

\section{Subjects \& Study Design}

All stroke patients presenting to Memorial Hermann Hospital Emergency Department (MHHED) and meeting published criteria for receiving intravenous tPA within 3 hours of symptom onset were screened. Subjects were at least 18 years of age.

All acute stroke patients have blood drawn upon arrival as part of standard of care. Patients who agreed to participate were consented for TEG analysis to be performed on residual blood. Within ten minutes post intravenous (IV) tPA bolus, a second blood draw was obtained. This time was chosen since, if TEG were eventually shown to predict success of clot lysis or subsequent bleeding, it would be soon enough to adjust the tPA dose.

Subjects were followed for 36 hours. Data collected included: age, race, gender, past medical history, current medications, pre-tPA CT, NIHSS pre-tPA and at 36 hours, TEG, glucose, hemoglobin, hematocrit, platelets, PT, INR, PTT and stroke subtype (Trial of Org 10172 in Acute Stroke Treatment (TOAST) criteria ${ }^{20}$ ).

\section{Controls}

Due to variability in the values of TEG parameters in healthy volunteers reported in the literature ${ }^{21-24}$ we obtained TEG samples from healthy volunteers at our center and compared our stroke patients to these contemporaneous samples measured on the same equipment and by the same personnel.

\section{Statistical Analysis}

Categorical variables were reported as frequency and percentages. Continuous variables with normal distributions were reported as mean (SD) while variables not having a normal distribution were reported as median (inter-quartile). The differences between the control group and our pre-tPA group were assessed using two-sample (independent) $t$ tests (or 
Wilcoxon rank sum tests) and $\chi^{2}$ tests as appropriate. The associations of all components of the pre-tPA TEG with age, gender, race, blood tests, home medications, medical history, stroke subtype, and NIH stroke scale in patients were assessed using linear regression. All factors associated with TEG values at $P<0.2$ in univariate analysis were tested into a multivariable regression analysis. The comparative histograms (with overlay of normal distribution curve) were presented to visualize the changes in TEG values before and after tPA treatment. Wilcoxon signed rank tests or paired t-tests were further performed to test differences in TEG values before and after tPA treatment. F tests or Levene's tests were used to test equality of variances of TEGs before and after tPA treatment. Pearson or Spearman correlation coefficient between total tPA dose and change in each TEG value after tPA treatment was calculated to detect the possible dose effects of tPA on changes in TEGs. All tests were conducted at 0.05 level of significance using SAS 9.2(Cary, NC).

\section{Sample Size}

Based on Ettinger's findings that 29-38\% of AIS patients are hypercoagulable compared to $12 \%$ of controls ${ }^{13}$, we estimated that we would need 50 subjects in each group to detect a statistically significant difference with $80 \%$ power at $5 \%$ level of significance. This sample size would also allow us to detect differences of 0.57 standard deviations for each of the TEG values with a power of $80 \%$.

\section{Blood Sampling \& Processing}

7-10 $\mathrm{ml}$ of whole blood was collected into a citrated tube by MHHED nurses upon the patient's arrival, prior to administration of tPA. The blood was held at room temperature and taken for processing within 2 hours of collection. $1 \mathrm{ml}$ of whole blood was placed into a kaolin vial and inverted 5 times for mixing. $340 \mu \mathrm{L}$ was then pipetted (polyethylene) into the disposable cup in the machine well. The citrate was then reversed with $20 \mu \mathrm{L}$ of $0.2 \mathrm{M}$ calcium chloride. It was gently mixed in the cup by pipette and the test was immediately run on a computerized TEG coagulation analyzer (Haemonetics Corp, Model 5000, Braintree Mass, USA@). Personnel who performed the testing were all trained on the procedure.

The following TEG values were documented at the completion of the test: $\mathrm{R}$ (minutes), $\mathrm{K}$ (minutes); Delta (R-SP, minutes); a Angle (degrees), maximal amplitude (MA, mm), G (dynes/cm2) and LY30 (percentage). R, K and Delta were available within 10 minutes of test initiation; Angle, MA and G within 20-30 minutes; and LY30 within 30 minutes.

The TEG machine was validated for quality assurance through daily quality control (QC) procedures using normal and abnormal controls (animal citrated whole blood) for calibration verification and operational checks. QCs were performed by technically trained research associates and a daily log maintained for quality assurance purposes.

\section{RESULTS}

From 11/2009 to 2/2011, 56 AIS subjects were enrolled in the study. 7 patients were not included in the analysis for the following reasons: Pre-tPA blood was not drawn (3), lack of venous access (1), processing of blood outside the 2 hour window (2), and neurological deficit resolved (1) making the total number of patients for analysis $49.47 \%$ were female 
and mean age was $66 \pm 16$. Average NIHSS score at presentation was $9.6 \pm 6.8$. Average time from symptom onset to blood draw was $106 \pm 43 \mathrm{~min}$. Post tPA samples were obtained in 30 of the 49 patients (post-tPA samples were initially not drawn in the first 15 subjects while awaiting IRB approval for the second blood draw). 4 subjects were eliminated from post tPA analysis due to processing error (3) and exceeding time window for sample collection (1). Average time from tPA bolus to $2^{\text {nd }}$ blood draw was 11 minutes.

Our study population was not significantly different from our control population in terms of gender, though there was a difference in age A AIS subjects mean age $=66 \pm 16$ vs control 55 \pm 13 ( $\mathrm{p}=0.0002)$,$\} . We did not access data on coronary artery disease, platelet count, or$ blood glucose in controls.

Univariate and multivariate linear regression models revealed that there was no relation between age and any of our TEG values (Table 1). Coronary artery disease, platelet count, and blood glucose level were associated with higher baseline $\mathrm{G}$ values, and coronary artery disease was associated with a longer R. MA negatively correlated with male gender, INR and platelet count, and a Angle was correlated with small vessel occulusion, hemoglobin and INR. The effect of hemoglobin on a Angle depended on the value of INR (significant interaction) and vice versa, and the effect of platelet count on MA also depended on the INR and vice versa.

Compared to controls, patients had shorter pre-tPA R, greater a Angle, shorter K, and shorter Delta. These changes indicate more rapid clot formation and strengthening in the stroke patients. Results of patients compared to controls are depicted in Table 2a and Figure 2.

In evaluating the distribution of pre-tPA $\mathrm{G}$ in our stroke patients, there appeared to be a subset of patients with very high values (Figure 3 ). Mean $G$ in local controls was $9.28 \pm 2.02$ (coefficient of variation $(\mathrm{CV})=0.22$ )(dotted vertical line). Mean $\mathrm{G}$ in stroke patients was $9.97 \pm 2.73\left(\mathrm{CV}=0.27\right.$ ). If we considered a $\mathrm{G}$ value of $11.3 \mathrm{kdynes} / \mathrm{cm}^{2}$ (highest control mean $+1 \mathrm{SD}$ ) as a threshold for supra-normal clot strength, 12 patients (24\%) exceeded this threshold and therefore represent a subpopulation of patients in which we might predict particularly strong, fibrin-platelet-rich clots.

Also depicted in Figure 3 is the range of $\mathrm{R}$ in our stroke patients, with the mean value in local controls $(5.96 \pm 1.67)(\mathrm{CV}=0.28)$ (horizontal dotted line). The 12 patients in the lower portion of the graph with low $\mathrm{R}$ demonstrate hypercoagulability by having more robust enzymatic coagulation (fast $\mathrm{R}$, intense thrombin generation).

Comparison between pre- and post-tPA TEG values are shown in Table $2 \mathrm{~b}$ and Figure 4 . There were major differences found in MA, G, and LY 30 reflecting breakdown of the fibrin clot matrix in post- tPA samples. There was also a difference in angle but no effect on average speed of clot initiation ( $\mathrm{R}$ or $\mathrm{K}$ ). Post -tPA TEG values also showed significantly greater variance compared to pre- tPA, especially in LY30 (Table $2 b$ ) indicating that the rate of clot formation and amount of fibrin dissolution was not uniformly suppressed by a weight based dosing of tPA in our cohort. For instance, note the large standard deviation in LY30 post-tPA (15.2-95.3) compared with pre-tPA $(0-0.4)(\mathrm{p}<0.0001)$. Patients with high pre- 
tPA-G, patients with low pre-tPA-R, and patients with both high pre-tPA-G and low pre-tPA $-\mathrm{R}$ did not differ from the respective counterparts in the changes of TEG values $(\Delta \mathrm{TEG})$ after tPA treatment $(\mathrm{P}>0.05)$ and all correlation coefficients between total tPA dose and $\triangle$ TEGs were $<0.1$, which indicated weak association between total tPA dose and $\triangle$ TEGs after tPA treatment. The graphic representation of the pre- and post-tPA distributions of MA, G, angle and LY30 is shown in Figure 4.

Based on our pre-tPA values of G and post-tPA LY30, we calculate that we will need data on 168 patients to determine if TEG can differentiate "red" from "white" clot, and predict recanalization post-tPA.

\section{DISCUSSION}

Our study is the first to evaluate TEG in AIS patients treated with tPA. Our major finding was that, compared to controls, many acute ischemic stroke patients ( 20 of 49 patients in our cohort) are hypercoagulable on various TEG parameters at the time they are being treated with tPA. Specifically, blood from our patients formed clots faster, and a subset of our patients had blood that formed clots with substantially stronger platelet-fibrin matrices. Although our patients were older than controls, this difference did not explain our results. The current literature demonstrates inconsistent results regarding the affect of aging on TEG values. Scarpelini et al 2009, ${ }^{21}$ found no effect of aging on TEG values and Roeloffzen et al $2010,{ }^{22}$ found that increasing age was associated with hypercoagulability, although the correlation coefficients were small ( $\mathrm{R}$ ranges $0.21-0.24$ for various TEG values). These results, combined with our own analysis of our local control population, lead us to conclude that there is a very small, if any, effect of aging on TEG values.. Our TEG values were affected by certain baseline variables, especially coronary artery disease, that were probably under-represented in our controls, and this may explain some of the elevations seen in our patients. However, the calculated effect of these variables, even if they were not present at all in our controls, could not explain all of the abnormal values seen in our patients.

We also found that TEG values are machine- and operator- dependent since there is variability among values reported in the literature for normal volunteers ${ }^{21,22}$ and also between literature controls and our local volunteers tested on our own equipment. While TEG results in our patients were more similar to our local controls than to literature controls, our patients were still abnormal in the same direction on the same measures when compared to all control populations, with all results indicating heightened coagulability.

We cannot say if the abnormalities found on TEG predated the stroke or were the results thereof. Similarly, we cannot say if the TEG values measured in venous blood were reflective of the clotting process that was simultaneously occurring in their arterial cerebral circulation causing their stroke. However, we did find substantial variability in clot strength among our patients as reflected in $G$ values. $G$ values represent the strength of the mature $\operatorname{clot}^{23-25}$. Barua et al found that only $13 \%$ of the total $\mathrm{G}$ is attributable to fibrin alone ${ }^{23}$; the remaining $87 \%$ is attributable to platelet-fibrin(ogen) interaction. Thus it is plausible that this variability in $\mathrm{G}$ reflects differential clot composition. Delta values are smaller in clots with extensive thrombin-generated fibrin matrices, since delta represents the intensity of the 
thrombin burst ${ }^{24}$. Recent studies demonstrated that clots extracted from stroke patients via endovascular mechanical clot extraction are heterogeneous with some that are "red" reflecting more fresh thrombin-generated RBC-fibrin rich clots, and others that are "white" or more rich in fibrin-platelet meshwork ${ }^{26}$. Furthermore, these clot subtypes might be differentiated on the basis of radiographic characteristics ${ }^{26}$. The next phase of our studies will include 168 patients to determine any relationship between our TEG values and radiographic findings and clot subtype.

Our patients' blood showed the expected response to tPA by having universally reduced clot strength in samples obtained 10 minutes after the tPA bolus. TEG appeared to reveal that tPA did not have a consistent, significant effect on the kinetics of the enzymatic portion of coagulation, but rather, had significant effects on the final strength and resulted in faster lysis of the formed clot. This was evidenced by differences in the pre and post IPA MA, G and LY 30, but no differences in R or K. Although a substantial effect on TEG values was seen in all patients tested after tPA, there was considerable variability in tPA's effect on clot lysis (LY30), suggesting that not all patients respond the same to an equivalent weightadjusted dose of tPA. IV tPA results in complete recanalization in only a minority of stroke patients within the first 2 hours ${ }^{18}$ and leads to symptomatic bleeding in $4 \%$ at our center. Jang et al. showed a differential response to tPA depending on the composition of clots in a rabbit model. ${ }^{27}$ Higher doses were needed to achieve recanalization of platelet-rich "white" clots, whereas RBC-rich "red" clots were lysed with lower doses. Based on the data in this first phase of our studies, we calculate that we will need 168 patients in the next phase to determine if pre- or post-tPA TEG values predict successful recanalization or bleeding.

We measured TEG 10 minutes after tPA bolus because if future analyses show that these TEG values predict response to tPA, there would still be time to alter the total dose given. Most of the values discussed in the present study may also be obtained from "rapid-TEG" within 15 minutes or less. ${ }^{28}$ However, it is important to note that the TEG values that we obtain do not directly measure the amount of clot lysis at the precise time the blood sample was obtained. Because the blood we collect is citrated, the TEG values we measure reflect the activity of tPA on the clot that occurs ex-vivo at the time the citrate is reversed. As mentioned, tPA appeared to have an immediate but variable effect on reducing the strength of such clot formation in all our patients, but we cannot be certain that these measurements exactly reflect the effect of tPA on the in-vivo clot.

We also carried out a platelet mapping assay consisting of percent platelet aggregation and inhibition on all pre-tPA blood samples, but did not include these data in the current analysis which focused on those TEG parameters that best represent the dynamics of clot formation and lysis. $43 \%$ of our patients were taking either aspirin or clopidogrel, but our analysis showed no interaction between anti-platelet therapy and any of the TEG parameters we studied.

Our study had many limitations. Our data were sufficient to confirm our three hypotheses and the results of a 37 year old study, and to establish TEG as a test that might be useful in helping us better understand the target of lytic therapy. However, as expected, the wide variance in post-tPA TEG values decreased the power for us to draw conclusions about any 
relationship between post-tPA TEG and clot lysis or outcome in this small sample of patients. We planned this first exploratory phase to gather data to determine the sample size needed for a larger study to determine if TEG can help predict response to thrombolytic therapy. Our study may be limited by the multiple operators carrying out TEG measurements. While we cannot exclude some imprecision based on this factor, we minimized quality concerns by careful QA. Again, larger numbers of patients will be needed to overcome any inherent imprecision in TEG measurements. We also cannot compare TEG to molecular markers of coagulation and fibrinolysis. The value of TEG can only be determined in a subsequent study which is powered to detect its ability to predict response to tPA. If such predictability is found, then it can be compared to other markers in this regard. Finally, we used controls that were not exactly matched to our patients, especially for age. However, for reasons described, we do not think these differences accounted for the hypercoagulability observed in our study.

In conclusion, our study demonstrates that many acute ischemic stroke patients are hypercoaguable. TEG values reflect variable clot subtype and response to tPA. Further study based on these data will determine if TEG is useful for measuring the dynamic aspects of clot formation and monitoring lytic therapy.

\section{Acknowledgments}

Supported by Tissue and Data Cores of National Institutes of Health 5P50NS044227-08. Haemonetics Corporation loaned a TEG coagulation analyzer $\odot$ Model 5000 and provided supplies.

\section{References}

1. Srinivasa V, Gilbertson LI, Bhavani-Shankar K. Thromboelastography: Where is it and where is it heading? International anesthesiology clinics. 2001; 39:35-49. [PubMed: 11285943]

2. Mallett SV, Cox DJ. Thrombelastography. British journal of anaesthesia. 1992; 69:307-313. [PubMed: 1389849]

3. Kang Y. Thromboelastography in liver transplantation. Seminars in thrombosis and hemostasis. 1995; 21 (Suppl 4):34-44. [PubMed: 8747686]

4. Kang YG, Martin DJ, Marquez J, Lewis JH, Bontempo FA, Shaw BW Jr, et al. Intraoperative changes in blood coagulation and thrombelastographic monitoring in liver transplantation. Anesthesia and analgesia. 1985; 64:888-896. [PubMed: 3896028]

5. Shore-Lesserson L, Manspeizer HE, DePerio M, Francis S, Vela-Cantos F, Ergin MA. Thromboelastography-guided transfusion algorithm reduces transfusions in complex cardiac surgery. Anesthesia and analgesia. 1999; 88:312-319. [PubMed: 9972747]

6. Kaufmann CR, Dwyer KM, Crews JD, Dols SJ, Trask AL. Usefulness of thrombelastography in assessment of trauma patient coagulation. Journal of trauma. 1997; 42:716-720. [PubMed: 9137263]

7. Steer PL. Anaesthetic management of a parturient with thrombocytopenia using thrombelastography and sonoclot analysis. Canadian journal of anaesthesia. 1993; 40:84-85. [PubMed: 8425253]

8. Steer PL, Finley BE, Blumenthal LA. Abruptio placentae and disseminated intravascular coagulation: Use of thrombelastography and sonoclot analysis. International journal of obstetric anesthesia. 1994; 3:229-233. [PubMed: 15636956]

9. Orlikowski CE, Rocke DA. Coagulation monitoring in the obstetric patient. International anesthesiology clinics. 1994; 32:173-191. [PubMed: 8063448]

10. Carroll RC, Craft RM, Whitaker GL, Snider CC, Kirby RK, Elder RF, et al. Thrombelastography monitoring of resistance to enoxaparin anticoagulation in thrombophilic pregnancy patients. Thromb Res. 2007; 120:367-70. [PubMed: 17150243] 
11. McCrath DJ, Cerboni E, Frumento RJ, Hirsh AL, Bennett-Guerreo E. Thromboelastography maximum amplitude predicts postoperative thrombotic complications including myocardial infarction. Anesthesia and analgesia. 2005; 100:1576-1583. [PubMed: 15920177]

12. Hadley GP, McGarr P, Mars M. The role of thromboelastography in the management of children with snake-bite in southern africa. Transactions of the Royal Society of Tropical Medicine and Hygiene. 1999; 93:177-179. [PubMed: 10450445]

13. Ettinger MG. Thromboelastographic studies in cerebral infarction. Stroke. 1974; 5:350-354. [PubMed: 4836538]

14. Feinberg WM, Bruck DC, Ring ME, Corrigan JJ Jr. Hemostatic markers in acute stroke. Stroke. 1989; 20:592-597. [PubMed: 2718198]

15. Feinberg WM, Erickson LP, Bruck D, Kittelson J. Hemostatic markers in acute ischemic stroke. Association with stroke type, severity, and outcome. Stroke. 1996; 27:1296-1300. [PubMed: 8711789]

16. Takano K, Yamaguchi T, Uchida K. Markers of a hypercoagulable state following acute ischemic stroke. Stroke. 1992; 23:194-198. [PubMed: 1561647]

17. Haapaniemi E, Tatlisumak T. Is d-dimer helpful in evaluating stroke patients? A systematic review. Acta neurologica Scandinavica. 2009; 119:141-150. [PubMed: 18705677]

18. Alexandrov AV, Demchuk AM, Burgin WS, Robinson DJ, Grotta JC. CLOTBUST Investigators. Ultrasound-enhanced thrombolysis for acute ischemic stroke: Phase i. Findings of the clotbust trial. Journal of neuroimaging. 2004; 14:113-117. [PubMed: 15095555]

19. Saqqur M, Tsivgoulis G, Molina CA, Demchuk AM, Siddiqui M, Alvarez-Sabín J, et al. Symptomatic intracerebral hemorrhage and recanalization after iv rt-pa: A multicenter study. Neurology. 2008; 71:1304-1312. [PubMed: 18753474]

20. Adams HP Jr, Bendixen BH, Kappelle LJ, Biller J, Love BB, Gordon DL, et al. Classification of subtype of acute ischemic stroke. Definitions for use in a multicenter clinical trial. Toast. Trial of org 10172 in acute stroke treatment. Stroke. 1993; 24:35-41. [PubMed: 7678184]

21. Scarpelini S, Rhind SG, Nascimento B, Tien H, Shek PN, Peng HT, et al. Normal range values for thromboelastography in healthy adult volunteers. Brazilian journal of medical and biological research. 2009; 42:1210-1217. [PubMed: 19882085]

22. Roeloffzen WW, Kluin-Nelemans HC, Mulder AB, Veeger NJ, Bosman L, de Wolf JT. In normal controls, both age and gender affect coagulability as measured by thrombelastography. Anesthesia and analgesia. 2010; 110:987-994. [PubMed: 20357143]

23. Barua RS, Sy F, Srikanth S, Huang G, Javed U, Buhari C, et al. Effects of cigarette smoke exposure on clot dynamics and fibrin structure: An ex vivo investigation. Arteriosclerosis, thrombosis, and vascular biology. 2010; 30:75-79.

24. Gonzalez E, Kashuk JL, Moore EE, Silliman CC. Differentiation of enzymatic from platelet hypercoagulability using the novel thrombelastography parameter delta (delta). Journal of surgical research. 2010; 163:96-101. [PubMed: 20605586]

25. Nielsen VG, Geary BT, Baird MS. Evaluation of the contribution of platelets to clot strength by thromboelastography in rabbits: The role of tissue factor and cytochalasin d. Anesthesia and analgesia. 2000; 91:35-39. [PubMed: 10866883]

26. Liebeskind DS, Sanossian N, Yong WH, Starkman S, Tsang MP, Moya AL, et al. Ct and mri early vessel signs reflect clot composition in acute stroke. Stroke. 2011; 42:1237-1243. [PubMed: 21393591]

27. Jang IK, Gold HK, Ziskind AA, Fallon JT, Holt RE, Leinbach RC, et al. Differential sensitivity of erythrocyte-rich and platelet-rich arterial thrombi to lysis with recombinant tissue-type plasminogen activator. A possible explanation for resistance to coronary thrombolysis. Circulation. 1989; 79:920-928. [PubMed: 2494006]

28. Jeger V, Zimmermann H, Exadaktylos AK. Can rapidteg accelerate the search for coagulopathies in the patient with multiple injuries? Journal of trauma. 2009; 66:1253-1257. [PubMed: 19359945] 


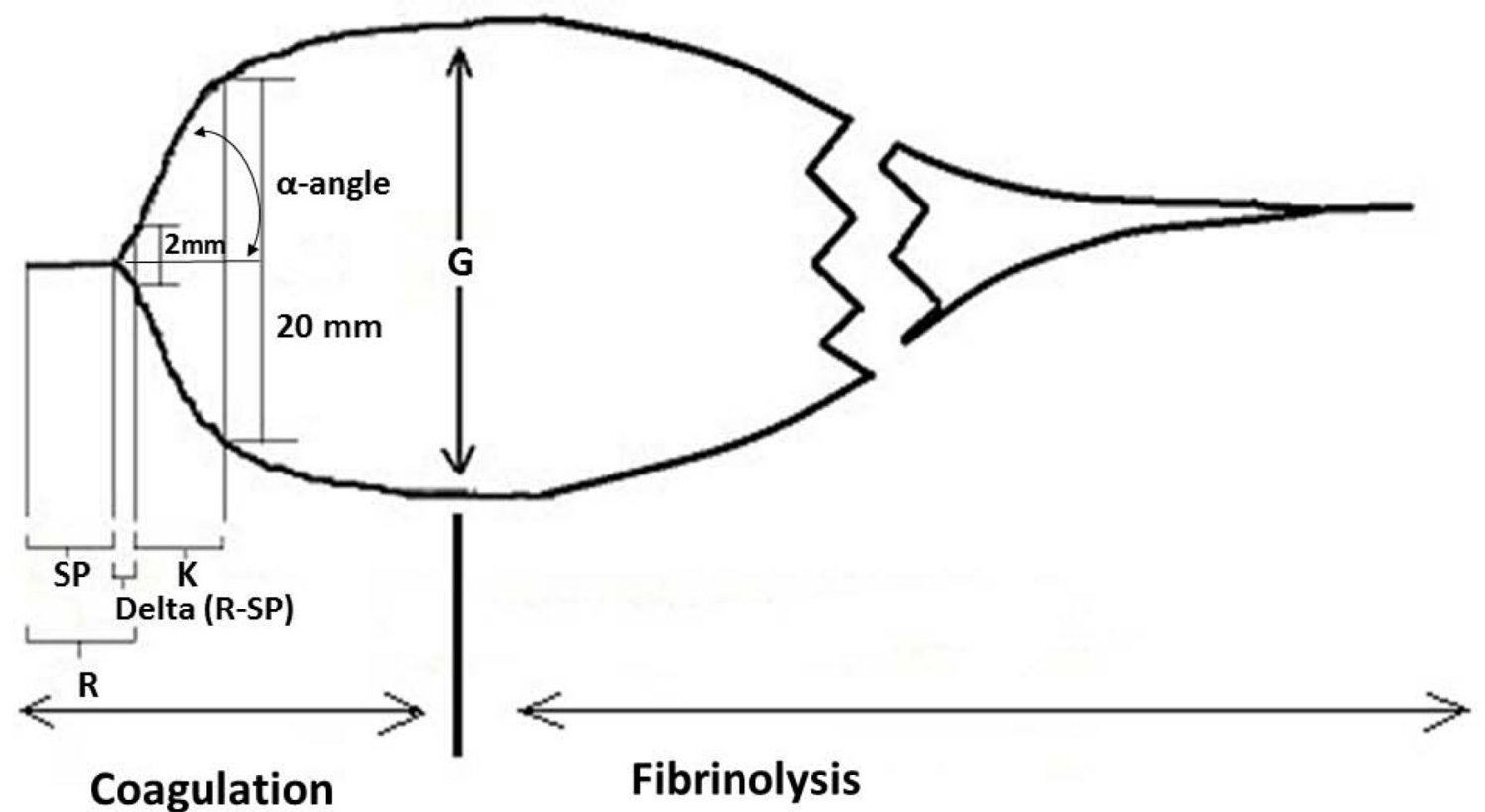

Figure 1.

A normal thromboelastograph (TEG) tracing. SP-initial fibrin formation. R-reaction time to clot formation. Delta- (R-SP) Thrombin burst. K- speed of clot strengthening. Angle- rate of initial clot strengthening. G-clot firmness. LY30-lysis as determined by clot strength measured 30 minutes after maximal amplitude of clot strength (MA) is reached. 


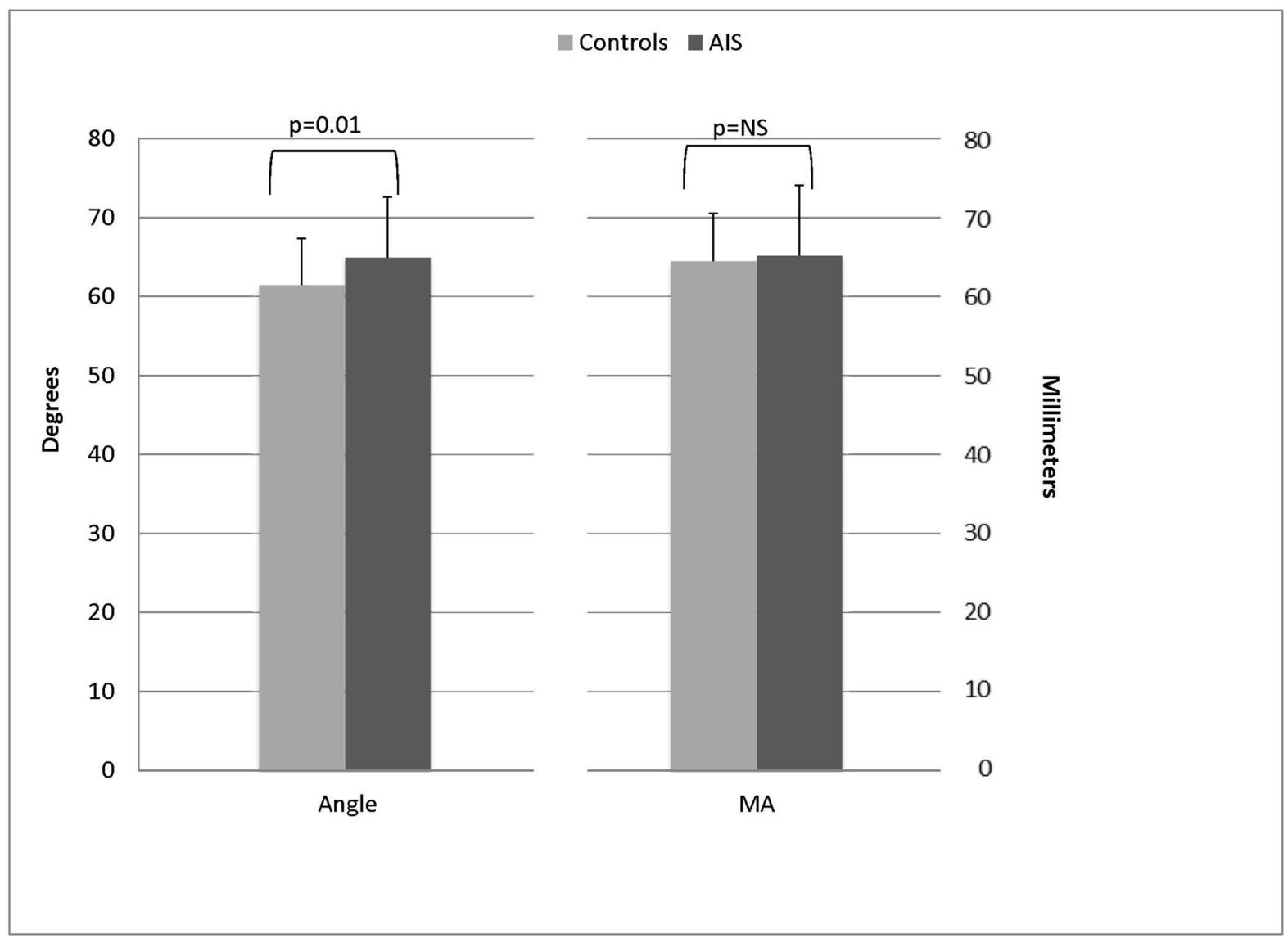

Int J Stroke. Author manuscript; available in PMC 2016 February 01. 


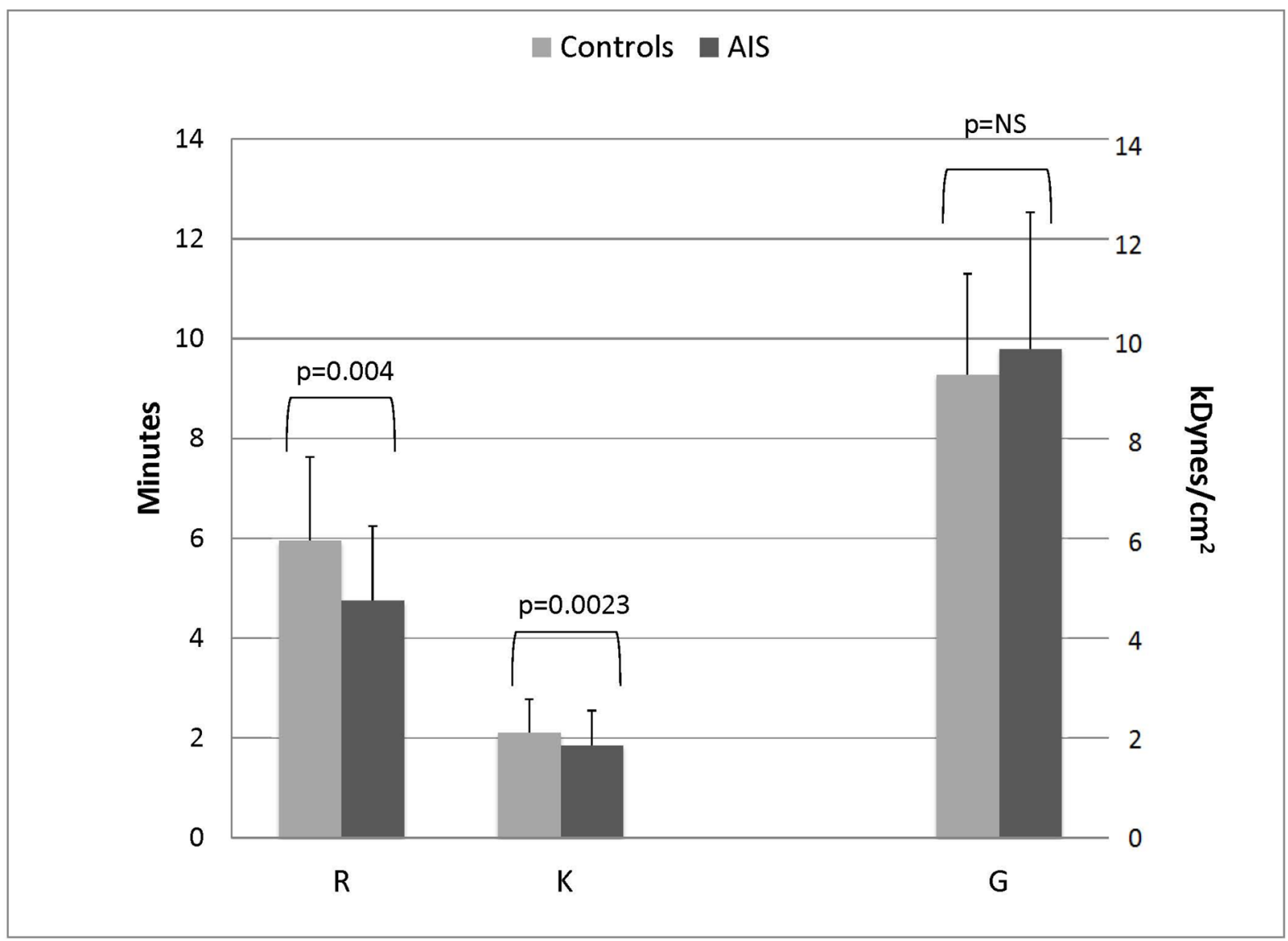

Int J Stroke. Author manuscript; available in PMC 2016 February 01. 


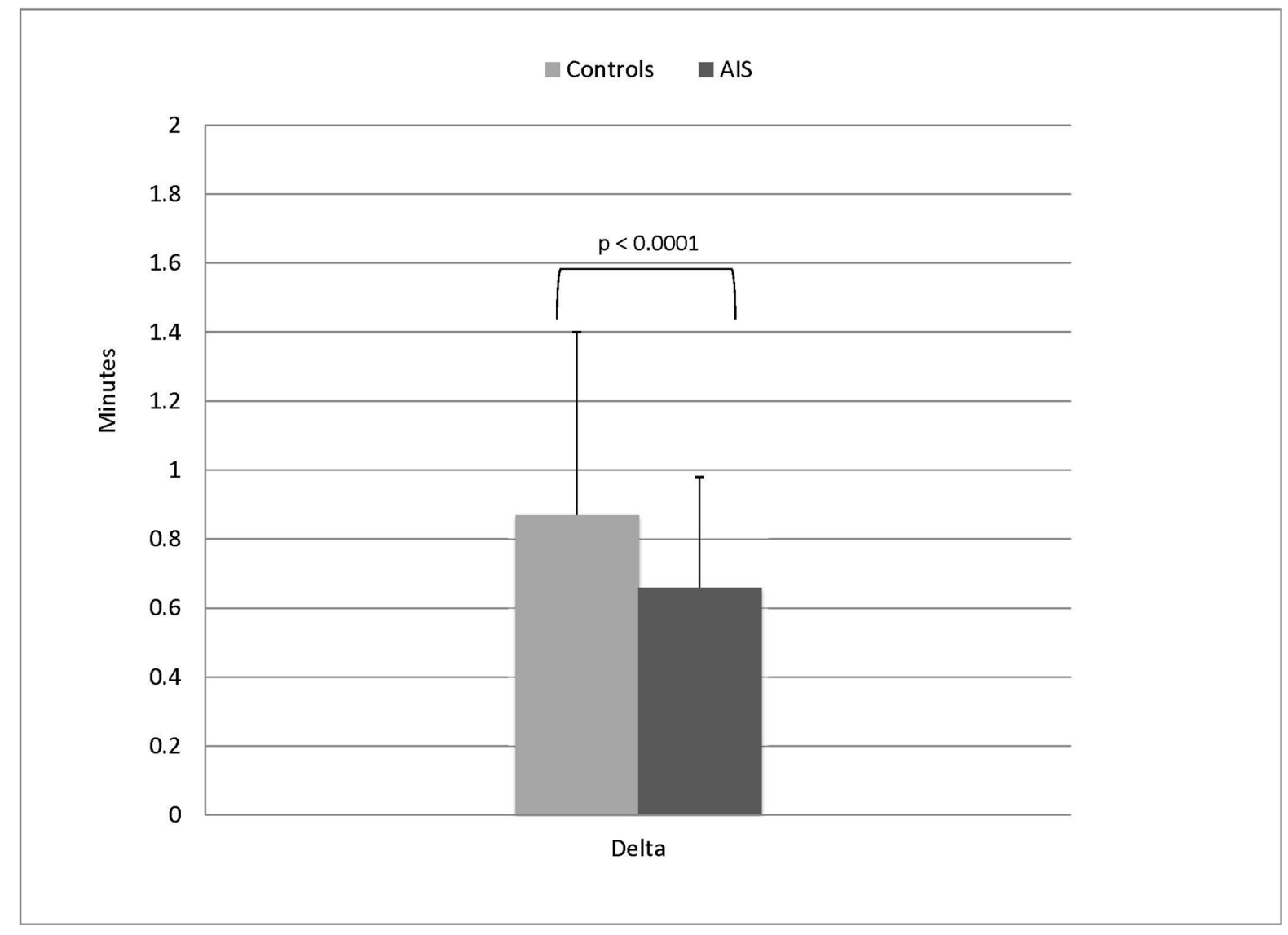

Figure 2.

Figure 2a. Angle and MA in AIS patients. Controls vs pre-TPA. NS=Not Significant Figure 2 b. R, K, and G in AIS patients. Controls vs pre-TPA. NS=Not significant.

Figure 2c. Delta in AIS patients. Controls vs pre-TPA. 


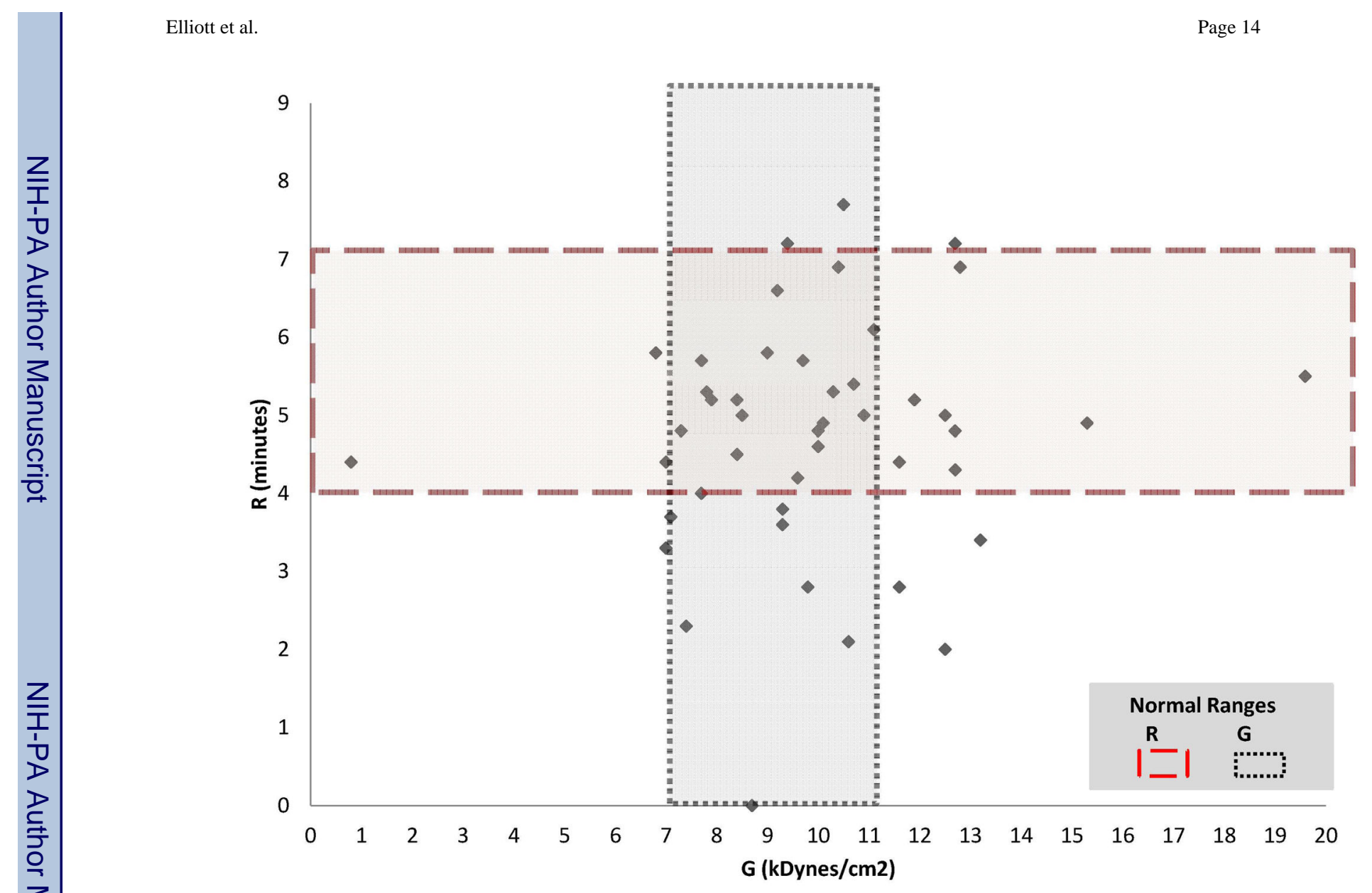

Figure 3.

$\mathrm{R}$ and $\mathrm{G}$ in pre-tPA patients. Vertical dotted line $=$ normal mean $\mathrm{G} \pm \mathrm{S}$.D. Horizontal dotted line $=$ normal mean $\mathrm{R} \pm \mathrm{S}$.D. Each represents a single patient. See text for interpretation. 

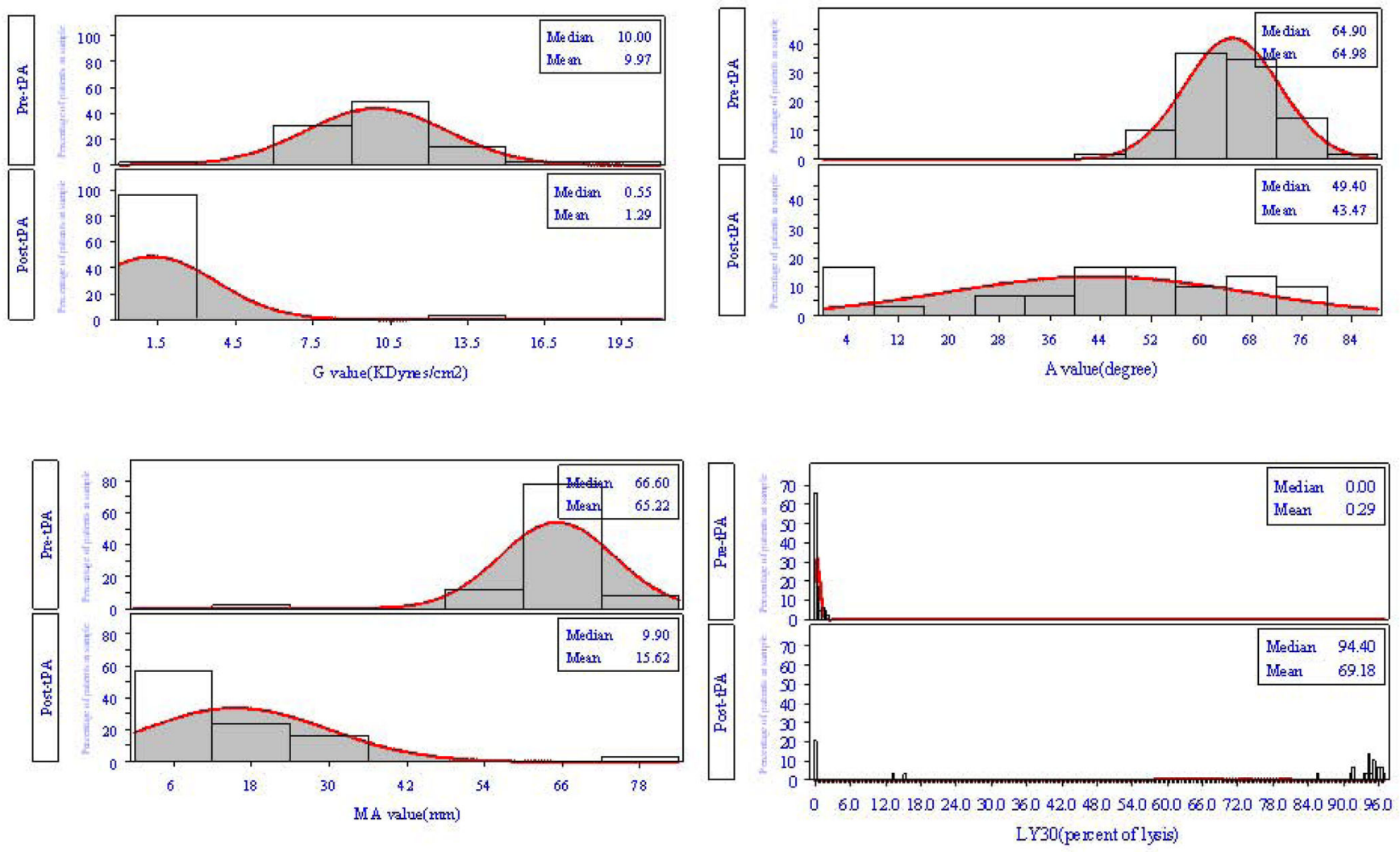

Figure 4.

G, A, MA, and LY30 in AIS patients. pre-tPA and post-tPA distribution curves. Y axis=percent of patients in sample. 
Table 1

Results of multivariable analysis for factors associated with TEGs

\begin{tabular}{|c|c|c|c|c|}
\hline TEG values & Variables & Coefficient & Standard error & $P$-value \\
\hline \multirow[t]{5}{*}{$\mathbf{G}$} & Male & -1.219 & 0.641 & 0.064 \\
\hline & Coronary Artery disease & 1.541 & 0.732 & 0.042 \\
\hline & Cardio embolic & -1.242 & 0.644 & 0.061 \\
\hline & Glucose & 0.015 & 0.005 & 0.008 \\
\hline & Platelet count & 0.014 & 0.006 & 0.025 \\
\hline \multirow[t]{3}{*}{$\mathbf{R}$} & Large artery atherosclerosis & 0.442 & 0.577 & 0.448 \\
\hline & Small vessel occlusion & -1.155 & 0.662 & 0.089 \\
\hline & Coronary Artery disease & 1.159 & 0.506 & 0.027 \\
\hline \multirow[t]{4}{*}{$\mathbf{A}$} & Small vessel occlusion & 5.699 & 2.818 & 0.049 \\
\hline & hemoglobin & 9.961 & 2.322 & $<.001$ \\
\hline & INR & 137.902 & 33.583 & $<.001$ \\
\hline & Hemoglobin* INR & -10.228 & 2.305 & $<.001$ \\
\hline \multirow[t]{6}{*}{ MA } & Male & -4.973 & 2.105 & 0.023 \\
\hline & Coronary Artery disease & 2.856 & 2.357 & 0.233 \\
\hline & Cardio embolic & -1.797 & 2.184 & 0.415 \\
\hline & Platelet count & -0.611 & 0.135 & $<.001$ \\
\hline & INR & -142.105 & 26.903 & $<.001$ \\
\hline & Platelet count*INR & 0.613 & 0.125 & $<.001$ \\
\hline \multirow[t]{2}{*}{$\mathbf{K}$} & Smoker & 0.462 & 0.238 & 0.059 \\
\hline & Platelet count & -0.003 & 0.001 & 0.027 \\
\hline
\end{tabular}




\section{Table 2}

TEG values in controls, pre-tPA and post-tPA stroke patients.

\begin{tabular}{|c|c|c|c|}
\hline \multicolumn{6}{|c|}{ Table 2a: TEG values in controls and pre-tPA stroke patients } \\
\hline & Control (N=49) & Pre-tPA-Stroke patients (N=49) & $\begin{array}{c}\text { P-values for difference of TEG between control and pre-tPA -Stroke } \\
\text { patients }\end{array}$ \\
\hline G & $9.28 \pm 2.02$ & $9.97 \pm 2.73$ & 0.1627 \\
\hline R & $5.96 \pm 1.67$ & $4.78 \pm 1.48$ & 0.0004 \\
\hline Angle & $61.46 \pm 5.89$ & $64.98 \pm 7.61$ & 0.0121 \\
\hline MA & $64.52 \pm 6.00$ & $65.21 \pm 8.85$ & 0.2259 \\
\hline K & $2.12 \pm 0.66$ & $1.65 \pm 0.69$ & 0.0023 \\
\hline Delta & $0.87 \pm 0.32$ & $0.66 \pm 0.53$ & 0.0002 \\
\hline LY30 & n/a & $0(0-0.4)$ & $\mathrm{n} / \mathrm{a}$ \\
\hline
\end{tabular}

\begin{tabular}{|c|c|c|c|c|}
\hline \multicolumn{2}{|c|}{ Table 2b: TEG values in pre-tPA and post-tPA stroke patients } \\
\hline & Pre-tPA-Stroke patients (N=49) & Post-tPA-Stroke patients (N=30) & $\begin{array}{c}\text { P-values for difference in } \\
\text { means of TEGs before } \\
\text { and after tPA }\end{array}$ & $\begin{array}{c}\text { P-values for difference in } \\
\text { variances of TEGs } \\
\text { before and after tPA }\end{array}$ \\
\hline G & $9.97 \pm 2.73$ & $1.29 \pm 2.45$ & $<.0001^{*}$ & 0.5483 \\
\hline R & $4.78 \pm 1.48$ & $127.58 \pm 342.3$ & 0.3068 & $<.0001^{*}$ \\
\hline Angle & $64.98 \pm 7.61$ & $43.47 \pm 23.56$ & $<.0001^{*}$ & $<.0001^{*}$ \\
\hline MA & $65.21 \pm 8.85$ & $15.62 \pm 14.23$ & $<.0001^{*}$ & 0.0035 \\
\hline K & $1.65 \pm 0.69$ & $11.71 \pm 16.04$ & 0.1563 & $<.0001^{*}$ \\
\hline Delta & $0.66 \pm 0.53$ & $1.42 \pm 3.42$ & 0.6297 & $<.0001^{*}$ \\
\hline LY30 & $0(0-0.4)$ & $94.4(15.2-95.3)$ & $<.0001^{*}$ & $<.0001^{*}$ \\
\hline
\end{tabular}

A significant difference in the variances pre- and post-tPA indicates a wider variability (significantly larger Standard Deviation) in the values after tPA compared to before.

Represents a significant change following administration of tPA.

${ }^{\dagger}$ Values for LY30 are reported as median (inter-quartile) because they did not follow a normal distribution. Table 2a: TEG values in controls and pre-tPA stroke patients 